\title{
PREPARATION AND CHARACTERIZATION OF METFORMIN LOADED STEARIC ACID COUPLED F127 NANOPARTICLES
}

\author{
VIPAN KUMAR KAMBOJ, PRABHAKAR KUMAR VERMA* \\ Department of Pharmaceutical Sciences, Maharshi Dayanand University, Rohtak - 124 001, Haryana, India. \\ Email: vermapk422@rediffmail.com
}

Received: 04 April 2018, Revised and Accepted: 27 April 2018

\section{ABSTRACT}

Objective: The objective of this study was to prepare and evaluate metformin nanoparticles (MN) using stearic acid-coupled F127 (SAF127) copolymer and polyvinyl alcohol by emulsion solvent evaporation technique.

Method: Metformin is the first-line drug for the treatment of type II diabetes mellitus belongs to Biopharmaceutical Classification System Class III. The prepared MN was characterized for particle size, polydispersity index (PDI), zeta potential, drug entrapment, percentage yield, in vitro drug release, and stability studies. The compatibility studies were performed by Fourier transform infrared (FTIR) and differential scanning calorimetry (DSC). The crystallographic and surface properties were studied by X-ray diffractometry and scanning electron microscopy, respectively.

Results: The mean particle diameter of prepared nanoparticles ranged from 207.8 to $977.64 \mathrm{~nm}$, PDI value ranged from 0.146 to 0.694 , and zeta potential ranged from -20.5 to $-6.97 \mathrm{mV}$. The drug entrapment efficiency of these nanoparticles varies between 18.81 to $69.01 \%$. The drug to SAF127 copolymer $(10 / 30 \mathrm{w} / \mathrm{w})$ ratio (MN3) showed optimum results. The MN3 had spherical morphology with semi-amorphous nature. The results of FTIR and DSC analysis showed that there was no significant interaction between drug and excipients. The prepared polymeric nanoparticles were stable at $5 \pm 3^{\circ} \mathrm{C}$ up to 3 months. In vitro release of drug from MN3 was $20.52 \%$ in the first $1 \mathrm{~h}$ and remaining drug was released up to $30 \mathrm{~h}$.

Conclusion: The results of this study confirmed the sustained drug release profile of metformin loaded SAF127 copolymer nanoparticles. These nanoparticles can be best stored up to 3 months.

Keywords: Metformin HCl, Nanoparticles, Pluronic F127, Polyvinyl alcohol, Stability studies.

(C) 2018 The Authors. Published by Innovare Academic Sciences Pvt Ltd. This is an open access article under the CC BY license (http://creativecommons. org/licenses/by/4. 0/) DOI: http://dx.doi.org/10.22159/ajpcr.2018.v11i8.26444

\section{INTRODUCTION}

Diabetes mellitus (DM) is one of the major incapacitating disorders spread in the worldwide population, contributing to huge financial and health loses. According to the studies conducted in India, highlighted that the prevalence of this metabolic dysfunction is high and increasing rapidly in the urban population due to the sedentary lifestyle, aging, nutrition, stress, and obesity [1,2]. It is discriminated by defects in insulin usage, either due to the autoimmune devastation of the insulinsecreting cells (Type I DM) or insulin resistance (Type II DM) [3]. The elevated blood glucose level of a diabetic patient is permanently untreatable, but this hyperglycemic condition can be managed by the daily dose of antidiabetic medications [2].

Metformin $\mathrm{HCl}$ is a highly water-soluble oral anti-hyperglycemic drug widely used as a drug of choice for the treatment of type II non-insulindependent DM [4]. Metformin improves the action of insulin at the cellular level without affecting its secretion [5]. It has low bioavailability (40-60\%) and short biological half-life (1.0-4.5 h) necessitate repeated administrations of doses (1.5-2.0 g/day) in two or three daily dose regiments to retain effective plasma concentrations [6-9]. The diabetic patients require life-long treatment and repeated administrations of doses reduce patient compliance and augment the frequency of side effects (e.g. diarrhea, abdominal discomfort, painful urination, and cramping) $[5,10]$. New formulation approaches for metformin are being pursued, which can improve bioavailability, reduced dose administration frequency, and improve patient compliance [11].

Nanotechnology-based oral drug delivery systems have become one of the fascinating research area and investigated for many drugs [2,12-15]. Nanoparticulate pharmaceutical formulations have properties of improved bioavailability, controlled, and prolonged drug release time with efficacy, safety and predicted therapeutic effect $[5,16]$. Various natural, synthetic, and semi-synthetic polymers have been investigated as a drug carrier to utilize the benefits of nanotechnology in formulations [6,16-21]. There is a persistent effort among the researchers to improve the formulation of metformin to attain optimal therapeutic response. The absorption and bioavailability of metformin might be improved by appropriate encapsulation of biocompatible and biodegradable polymeric systems.

In the current investigation, metformin loaded SAF127 nanoparticles were prepared by emulsion solvent evaporation technique. The prepared nanoparticles were optimized for particle size, PDI, zeta potential, entrapment efficiency, and percentage yield. The stability studies of $\mathrm{MN}$ were performed at $5 \pm 3$ and $25^{\circ} \mathrm{C}$ for 3 months.

\section{MATERIALS AND METHODS}

\section{Materials}

The pharmaceutical grade metformin $\mathrm{HCl}$ was obtained as a gift sample from Mankind Pharma, Paonta Sahib, India. Polyvinyl alcohol (PVA), stearic acid (SA), and Pluronic F127 have been procured from SigmaAldrich, India. Other chemical and solvents used were of analytical grade and have been purchased from Molychem, Mumbai.

\section{Preparation of SAF127 copolymer}

SAF127 copolymer was synthesized according to the reported method [15]. Pluronic F127 $10 \mathrm{~g}$ and SA $10 \mathrm{~g}$ were added in $100 \mathrm{ml}$ round bottom flask and the mixture was heated with constant stirring to yield a well-mixed molten phase and reacted at $155^{\circ} \mathrm{C}$ for $5.5 \mathrm{~h}$. The unreacted SA was removed by adding molten mixture into the 
ethyl acetate/petroleum ether 1:1 (v/v). The SAF127 copolymer was recovered by filtration and the organic solvent was evaporated at room temperature and further dried under vacuum for $24 \mathrm{~h}$. The structure of synthesized copolymer was confirmed by the spectrum of Fourier transform infrared (FTIR) (1-206-0280; Software: OPUS-7.2.139.1294) and ${ }^{1} \mathrm{H}$ nuclear magnetic resonance ${ }^{1} \mathrm{H}$ NMR; (Bruker Model Avance II 400; $400 \mathrm{MHz}$ ) spectroscopy.

\section{Preparation of metformin-Loaded SAF127 nanoparticles}

Metformin nanoparticles (MN) were formulated through the emulsion solvent evaporation technique. Accurately weighed SAF127 copolymer was dissolved in $10 \mathrm{~mL}$ of dichloromethane. Metformin was mixed with the aqueous solution of $15 \mathrm{ml}$ of PVA $(0.5 \% \mathrm{w} / \mathrm{v})$. SAF127 copolymer containing organic phase was added to the aqueous solution of metformin (w/o mixture). The obtained mixture was emulsified using probe sonication (Sonic Vibra-Cell"' VCX 750w) for 2 minutes to produce a water-in-oil-in-water emulsion. The dichloromethane was vaporized using a rotary evaporator (IKA ${ }^{\circledR}$ RV 10, BS96, Germany). The nanoparticles in residual solution were collected by centrifugation (14000 rpm; $30 \mathrm{~min}$; Remi, India) followed by lyophilization [11].

\section{Characterization of prepared MN}

\section{Particle size, PDI, and zeta potential}

The nanoparticle samples were suspended in Milli-Q water and screened for particle size, PDI, and zeta potential at $25^{\circ} \mathrm{C}$ by Zetasizer (Nano-ZS90, Malvern Instruments, UK). The disposable cuvettes were used for sample analysis. The results were reported as the mean \pm standard deviation for three replicates [22].

\section{Entrapment efficiency and percentage yield}

The supernatant liquid was collected during preparation and amount of metformin present in it was compared with the total amount of metformin used in the formulation of a batch. The non-encapsulated drug in supernatant aqueous phase was ascertained at $232 \mathrm{~nm}$ using UV-Vis spectrophotometer (Lab India-3000+). The drug entrapment efficiency (\%) and yield (\%) were calculated using Equation 1 and 2, respectively.

$$
\begin{aligned}
& \text { Entrapment efficiency }(\%) \\
& =\frac{\text { Amount of metformin in nanoparticles }}{\text { Amount of metformin used in the formulation }} \times 100 \\
& \text { Yield }(\%)=\frac{\text { Total nanoparticles weight }}{\text { Total solid weigh }} \times 100
\end{aligned}
$$

\section{FTIR spectrophotometer}

FTIR spectroscopy is a simple, sensitive, and multidimensional analytical tool used for recognizing changes in functional groups of pharmaceuticals [19]. FTIR spectra of SAF127, PVA, pure metformin, physical mixture, and MN were taken separately by KBr Pellet method using Bruker (1-206-0280; software: OPUS-7.2.139.1294, MENTOR) spectrophotometer. The spectra of samples were recorded in the range of $4000-400 \mathrm{~cm}^{-1}$.

\section{Differential scanning calorimetric (DSC) analysis}

Selected samples were examined for thermotropic properties using DSC Q10 V9.9 Build 303 (Waters, India) instrument. The DSC instrument was calibrated using Indium as standard. Accurately weighed $2 \mathrm{mg}$ samples were sealed in standard aluminum pans and screened between 30 to $300^{\circ} \mathrm{C}$ with a heating rate of $10^{\circ} \mathrm{C} / \mathrm{min}$ under the nitrogen environment (60 $\mathrm{ml} / \mathrm{min}$ ). The empty aluminum pan was used as a reference.

\section{X-ray diffractometry (XRD) analysis}

The XRD analysis was carried out for all the samples used in the FTIR study to know the crystalline and amorphous characteristics. XRD analysis was performed by Rigaku Miniflex-600 diffractometer. The instrument uses $\mathrm{CuK} \alpha$ radiation produced at $30 \mathrm{kV}$ with $15 \mathrm{~mA}$ current. The diffraction pattern was recorded over a $2 \theta$ with angular range of 10 to 70 .
Surface morphology study

The surface morphology of the optimized MN batch was studied by field emission scanning electron microscopy (FE-SEM) (JEOL, JSM-7600F, Japan). The gold coating on nanoparticles was performed using the ionsputtering machine and vacuum dried before the examination.

\section{In vitro drug release studies}

In vitro release studies were accomplished for optimized batch MN3 and pure metformin by dialysis sac method [6]. Accurately weighed samples were placed in dialysis bags (12-14 kDa cut-off, HiMedia, India) and tied with dialysis thread. The dialysis bags containing metformin nanosuspension and pure metformin suspension were immersed separately in a conical flask with $150 \mathrm{ml}$ of phosphate buffer solution $(0.1 \mathrm{M})$ with $\mathrm{pH}$ 7.4. The conical flask was stirred at $100 \mathrm{rpm}$, and the temperature maintained at $37 \pm 5^{\circ} \mathrm{C}$. At predetermined intervals, the aliquot of $1 \mathrm{ml}$ was taken from the conical flask and replenished with an equal amount of fresh phosphate buffer and the assay was performed using UV-Vis spectrophotometer (Lab India $3000^{+}$) at $232 \mathrm{~nm}$.

\section{Stability study}

The stability studies of MN3 were performed on two temperatures $\left(5 \pm 3\right.$ and $\left.25^{\circ} \mathrm{C}\right)$. The aliquot of MN suspension samples was withdrawn after completion of $1^{\text {st }}$ and $3^{\text {rd }}$ months. These samples were examined for any possible change in particle size, PDI, zeta potential, entrapment efficiency, and color of nanosuspension.

\section{RESULTS AND DISCUSSION}

\section{Characterization of SAF127 copolymer}

The esterification reaction between the carboxyl group of SA and hydroxyl group of Pluronic F127 yield SAF127 copolymer. The successful coupling of SA with Pluronic F127 was confirmed by FTIR spectra of copolymer having a band around $1728.96 \mathrm{~cm}^{-1}$ which were assigned to the stretching vibration of $\mathrm{C}=0$ ester bond. Major features of ${ }^{1} \mathrm{H}$ NMR spectra of SAF127 are enlisted in Table 1.

\section{Preparation of polymeric nanoparticles}

The polymeric nanoparticles of metformin were prepared by an emulsion solvent evaporation method, and metformin to copolymer amount was changed to find the best working ratio. The MN was screened for particle size, PDI, zeta potential, entrapment efficiency, and percentage yield and results are shown in Table 2. Particle size analysis and zeta potential of the best batch (MN3) are shown in Fig. 1.

The nanoparticles size was found to be minimum (207.8 \pm 4.09 ) for MN3 whereas maximum $(977.64 \pm 5.43 \mathrm{~nm})$ for the MN8 batch. The PDI value of batch MN3 and MN8 was found to be $0.146 \pm 0.038$ and $0.694 \pm 0.053$, respectively. The values of zeta potential for MN3 were $-20.5 \pm 0.14 \mathrm{mV}$, which support the stability of nanoparticles during storage phase. Drug entrapment efficiency and percentage yield of MN3 were observed as $69.01 \pm 3.48$ and $82.14 \pm 4.31 \%$, respectively.

The nano-sized particles having high zeta potential irrespective of charge type (negative or positive) and low PDI value imparted the stability during storage time [23,24]. A fixed concentration of PVA used as a surfactant which stabilizes the zeta potential of nanoparticles. Higher the entrapment efficiency of the drug in nanoparticles, the loss of drug during formulation process get reduced and nanoparticle yield increased $[24,25]$. The nanoparticles carrying a higher percentage of

Table 1: Major features of ${ }^{1} \mathrm{H}$ NMR spectra of SAF127

\begin{tabular}{ll}
\hline $\boldsymbol{\delta}(\mathbf{p p m})$ & Assign \\
\hline $\mathrm{CH}_{2}-\mathrm{O}$ in PEO & $3.67-3.65$ \\
$\mathrm{CH}_{2} \mathrm{CH}_{2}-\mathrm{O}$ in PEO & $2.37-2.35$ \\
$\mathrm{CH}_{2} \mathrm{CH}_{\left(\mathrm{CH}_{3}\right)-\mathrm{O} \text { in PEO }}$ & $1.65-1.63$ \\
$\mathrm{CH}_{2} \mathrm{CH}\left(\mathrm{CH}_{3}\right)-\mathrm{O}$ in PEO & $1.31-1.28$ \\
$\mathrm{CH}_{2}$ in SA & $1.18-1.15$ \\
\hline
\end{tabular}


the drug can achieve the therapeutic effect in a small dose and final size of dosage form get reduced which can be administered with more ease to young and geriatric patients.

\section{FTIR studies}

Compatibility of the drug to excipients was screened by FTIR and results are compiled in Fig. 2. The characteristic peaks of metformin were detected between 3310-3442, 3292, and 3000-3174 $\mathrm{cm}^{-1}$ relative to the stretching vibration of primary $\mathrm{NH}$, banding vibration of imines group (NH), and stretching vibration of secondary $\mathrm{NH}$. The characteristic bands at 1622 , and $1561 \mathrm{~cm}^{-1}$ were assigned to $\mathrm{C}-\mathrm{N}$ stretching vibrations. Frequently, the peak intensity of these vibrations is reduced due to the presence of hydrogen bonding [11]. In metformin loaded SAF127 nanoparticles, all the characteristic peaks of metformin were present with slightly reduced intensity. This indicates that the drug and excipients did not interact significantly with each other.

\section{DSC analysis}

DSC thermogram of SAF127 copolymer shows an endothermic peak at $50.23^{\circ} \mathrm{C}$, and glass transition was detected at $126.0^{\circ} \mathrm{C}$. The endothermic peak due to PVA was observed at $225.0^{\circ} \mathrm{C}$. The sharp endothermic peak due to pure metformin was obtained at $236.35^{\circ} \mathrm{C}$. All the peaks due to drug, and excipients were present in the physical mixture and MN3 (Fig. 3). On the basis of thermal behavior, drug, and excipients used in this investigation were found to be compatible with each other.
XRD studies

XRD analysis suggested that the metformin was in the crystalline state whereas SAF127 copolymer, PVA, and MN3 were in a semi-amorphous state (Fig. 4). All the significant peaks of drug, and SAF127 copolymer were present in the physical mixture. During the fabrication of nanoparticles, crystalline nature of metformin changes into partial amorphous nature. In MN3, fewer peaks were present with reduced intensities. The molecules of pure metformin were well arranged in crystal lattice whereas in the MN3 crystal lattice pattern gets distorted and acquire semi-amorphous nature. These results revealed that the drug was encapsulated inside the polymeric system and change in phase was observed [26].

\section{In vitro drug release studies}

The in vitro drug release studies of MN3 showed controlled drug release profile as compared to pure metformin. The $100 \%$ drug from pure metformin was released within $1 \mathrm{~h}$ whereas, only $20.52 \%$ drug was released in first $1 \mathrm{~h}$, and $100 \%$ metformin was released further for $30 \mathrm{~h}$ from MN3. The drug release profile of MN3 shows a sustained release pattern with the passage of time. Same drug release pattern was reported earlier [17]. The encapsulated drug released slowly with the eruption of polymeric layers and completed up to $30 \mathrm{~h}$. The cumulative drug release profile of MN3 and pure metformin is shown in Fig. 5.

\section{Morphological studies}

The nanoparticles were found to be spherical in shape by FE-SEM. The long crystals of metformin $\mathrm{HCl}$ were not visible in MN3. The

Table 2: Particle size, PDI, and zeta potential of batches

\begin{tabular}{lllllll}
\hline Batch & Drug/copolymer $(\mathbf{m g})$ & Particle size $(\mathbf{n m})$ & PDI & Zeta potential $(\mathbf{m V})$ & Entrapment Efficiency (\%) & Percentage yield \\
\hline MN1 & $10 / 10$ & $553.40 \pm 3.84$ & $0.423 \pm 0.03$ & $-12.41 \pm 4.53$ & $52.36 \pm 4.54$ & $70.68 \pm 5.24$ \\
MN2 & $10 / 20$ & $415.60 \pm 3.91$ & $0.244 \pm 0.012$ & $-17.46 \pm 1.15$ & $55.29 \pm 3.87$ & $71.49 \pm 4.18$ \\
MN3 & $10 / 30$ & $207.80 \pm 4.09$ & $0.146 \pm 0.038$ & $-20.50 \pm 0.45$ & $69.01 \pm 3.48$ & $82.14 \pm 4.31$ \\
MN4 & $10 / 40$ & $717.36 \pm 3.65$ & $0.767 \pm 0.007$ & $-11.93 \pm 1.15$ & $41.47 \pm 4.91$ & $68.82 \pm 3.63$ \\
MN5 & $10 / 50$ & $811.13 \pm 4.33$ & $0.584 \pm 0.004$ & $-8.73 \pm 0.90$ & $23.11 \pm 3.65$ & $58.73 \pm 3.88$ \\
MN6 & 20/10 & $613.60 \pm 4.81$ & $0.356 \pm 0.033$ & $-13.84 \pm 1.25$ & $38.46 \pm 4.52$ & $63.34 \pm 4.15$ \\
MN7 & $30 / 10$ & $894.36 \pm 4.63$ & $0.648 \pm 0.045$ & $-10.24 \pm 1.53$ & $26.53 \pm 4.64$ & $54.84 \pm 5.21$ \\
MN8 & $40 / 10$ & $977.64 \pm 5.43$ & $0.694 \pm 0.053$ & $-6.97 \pm 1.9$ & $18.81 \pm 5.42$ & $48.75 \pm 4.83$ \\
\hline
\end{tabular}

$\mathrm{n}=3$, mean values \pm SD. SD: Standard deviation, PDI: Polydispersity index

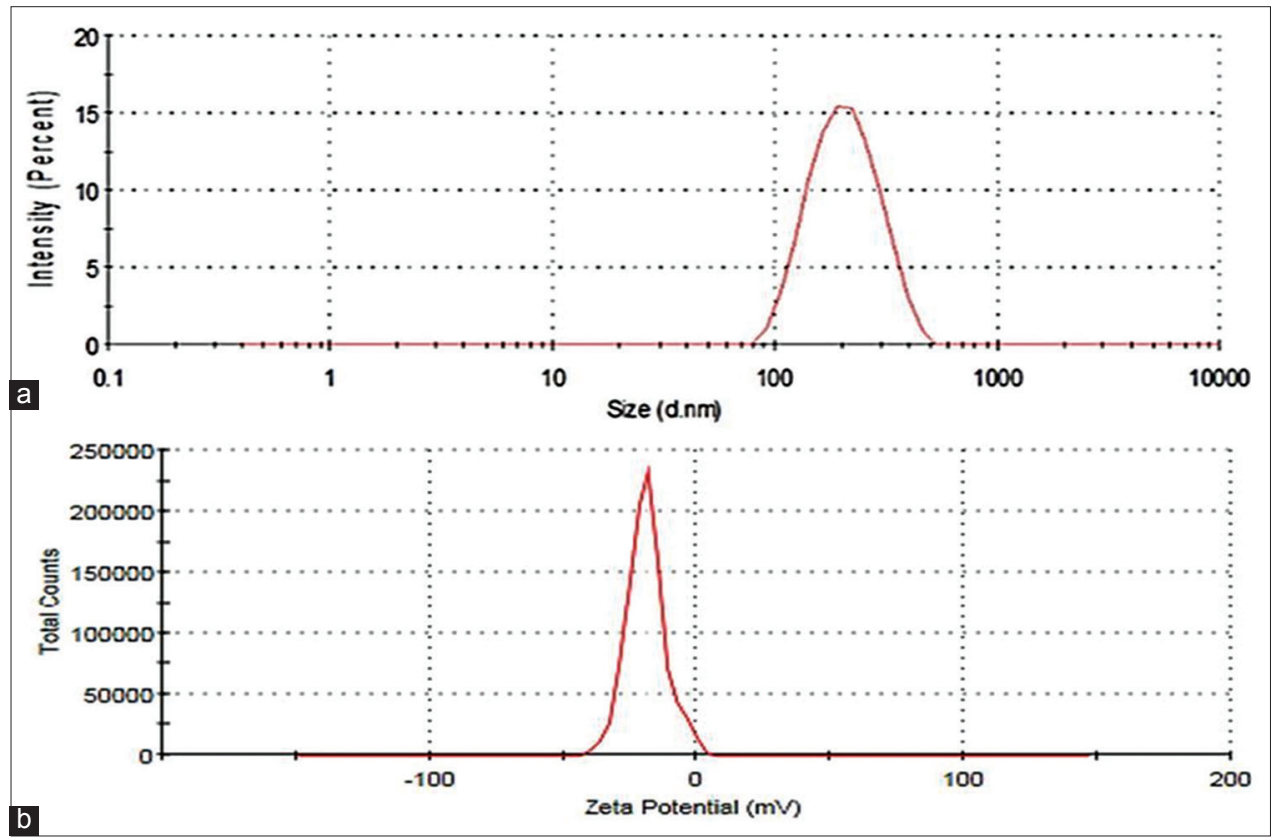

Fig. 1: (a) PSA and (b) zeta potential of MN3 


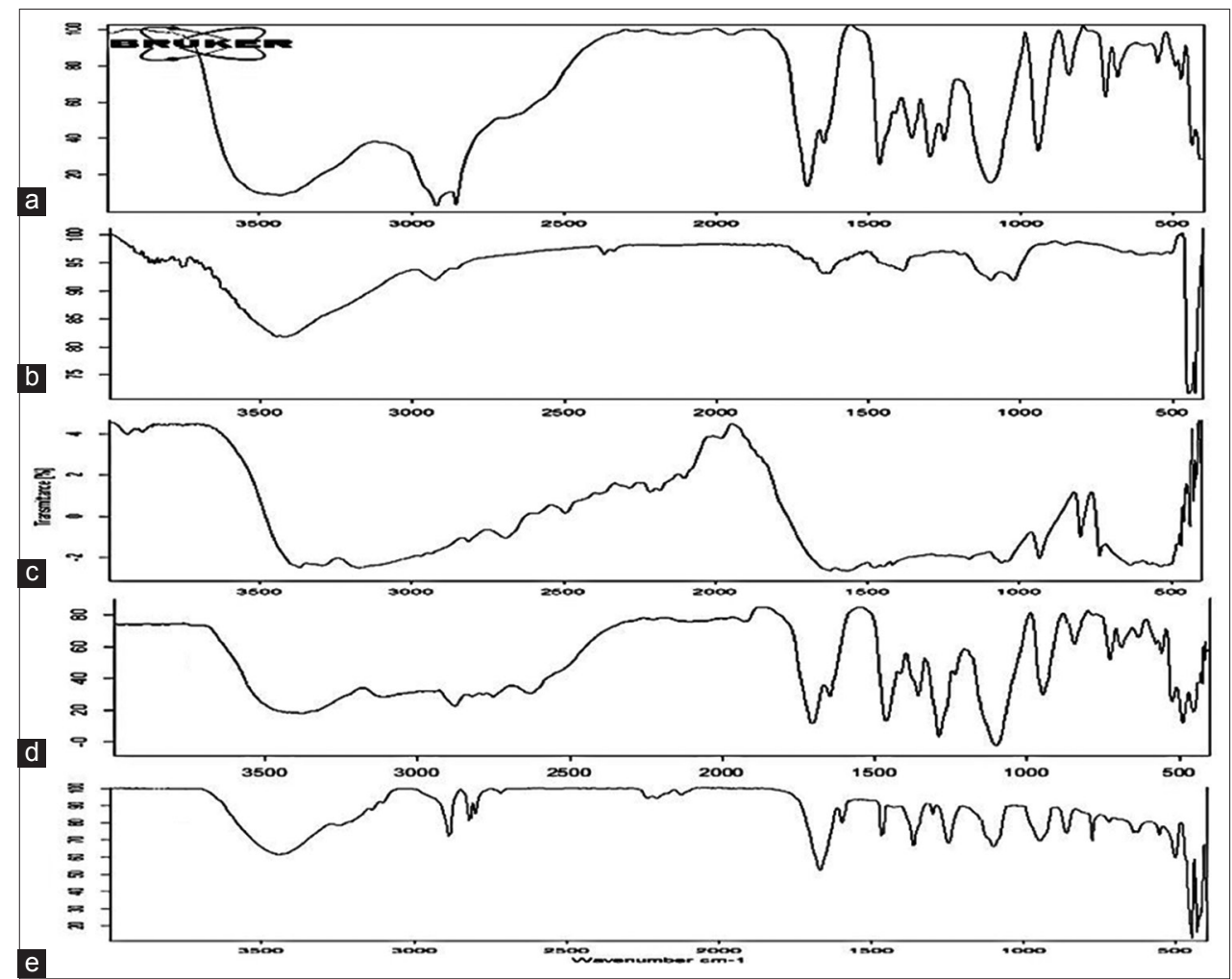

Fig. 2: Fourier transform infrared spectra of (a) SAF127 copolymer, (b) polyvinyl alcohol, (c) metformin, (d) physical mixture, and (e) MN3

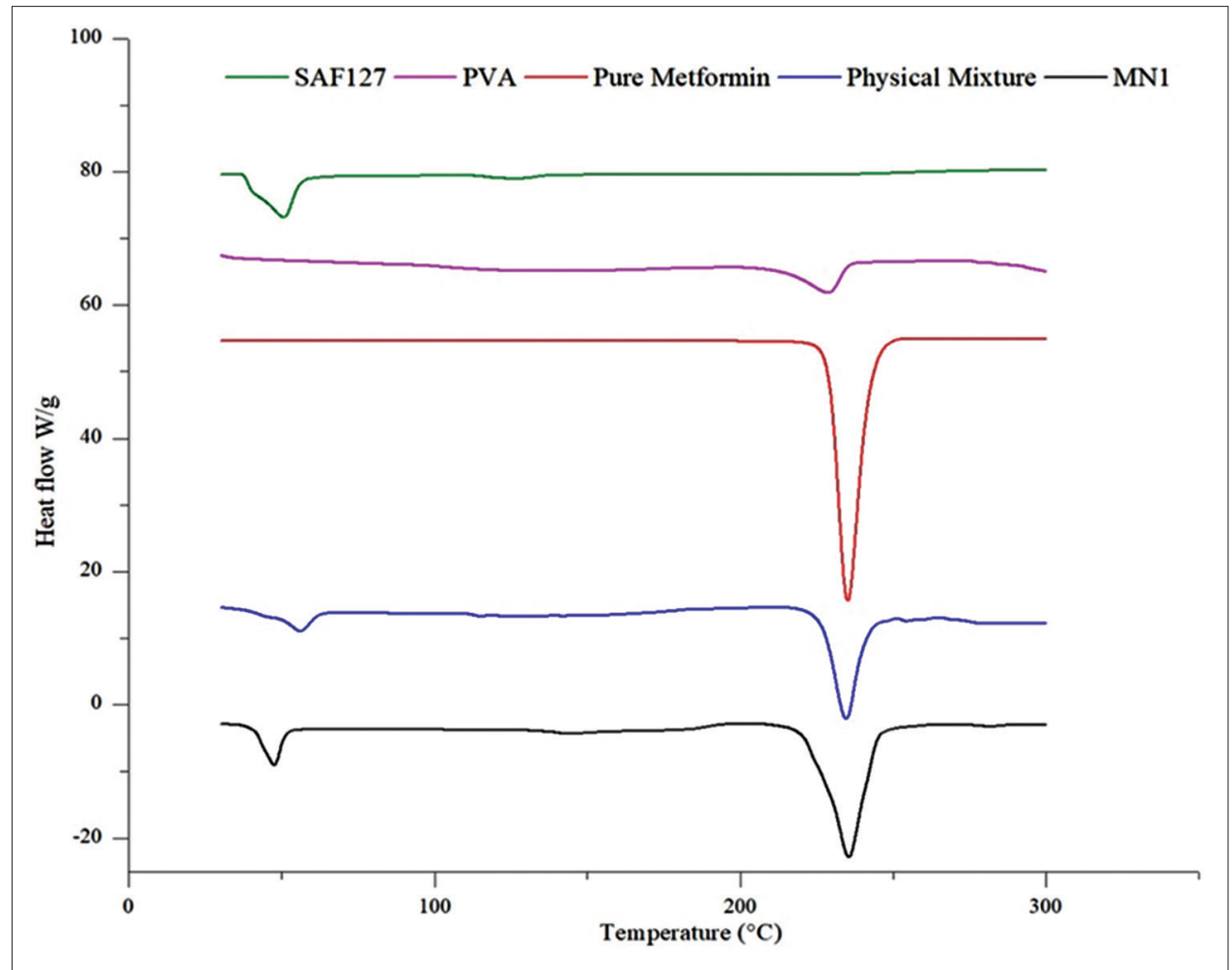

Fig. 3: Differential scanning calorimetry thermogram of SAF127 copolymer, polyvinyl alcohol, pure metformin, physical mixture, and MN3

nanoparticles size seen under FE-SEM was smaller than the size obtained by PSA. The samples screened by PSA were dissolved in Milli-Q water whereas anhydrated solid nanoparticles sample were tested by FE-SEM. In general, particles size found to be lesser in anhydrous solids as compared to hydrated solids [27].

\section{Stability studies}

Three months stability studies were performed for MN3 at two temperatures $\left(5 \pm 3\right.$ and $\left.25^{\circ} \mathrm{C}\right)$, and results are enlisted in Table 3 . The MN3 suspensions stored at both temperature remains carry particles size $<240 \mathrm{~nm}$. PDI, zeta potential, and entrapment efficiency were 
Table 3: Stability studies of MN3

\begin{tabular}{lllll}
\hline Storage condition & Particle size (nm) & PDI & Zeta potential $(\mathbf{m V})$ & Entrapment efficiency (\%) \\
\hline Fresh MN3 & $207.8 \pm 4.09$ & $0.146 \pm 0.03$ & $-20.5 \pm 0.45$ & $69.01 \pm 3.48$ \\
1 month $\left(5 \pm 3^{\circ} \mathrm{C}\right)$ & $214.4 \pm 5.14$ & $0.150 \pm 0.04$ & $-20.10 \pm 0.52$ & $68.35 \pm 4.43$ \\
3 months $\left(5 \pm 3^{\circ} \mathrm{C}\right)$ & $219.5 \pm 4.23$ & $0.161 \pm 0.04$ & $-19.51 \pm 0.42$ & $67.33 \pm 5.1$ \\
1 month $\left(25^{\circ} \mathrm{C}\right)$ & $222.4 \pm 4.64$ & $0.158 \pm 0.05$ & $-19.82 \pm 0.45$ & $68.43 \pm 5.2$ \\
3 months $\left(25^{\circ} \mathrm{C}\right)$ & $239.7 \pm 5.83$ & $0.226 \pm 0.06$ & $-17.57 \pm 0.55$ & $65.35 \pm 4.6$ \\
\hline
\end{tabular}

$\mathrm{n}=3$, mean values \pm SD. SD: Standard deviation, PDI: Polydispersity index

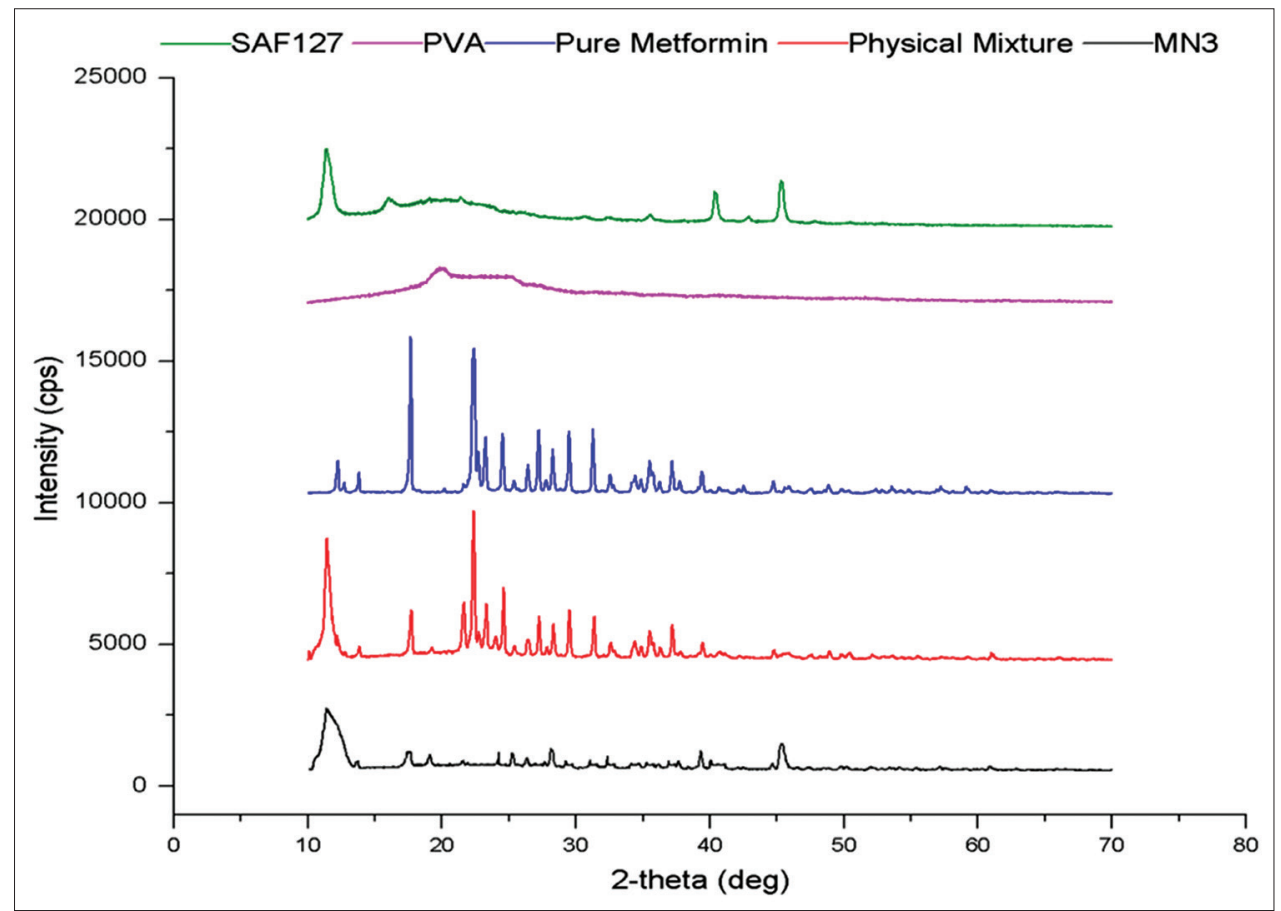

Fig. 4: X-ray diffractometer patterns of SAF127, polyvinyl alcohol, pure metformin, physical mixture, and MN3

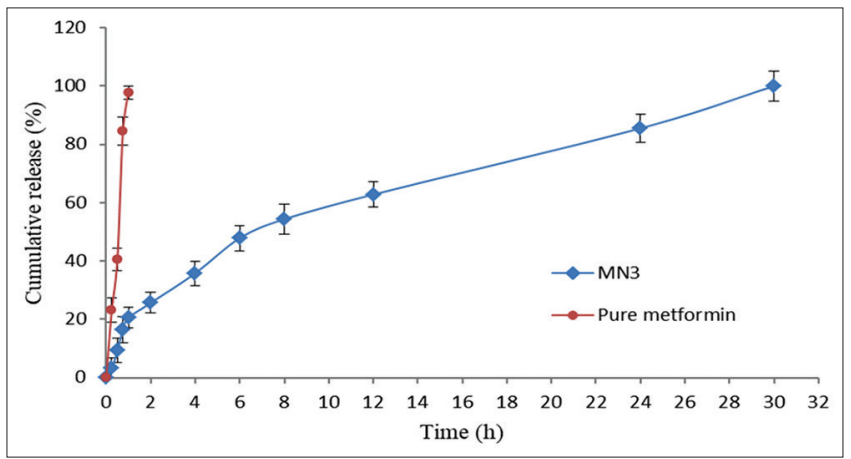

Fig. 5: Cumulative drug release from MN3 and pure metformin

changed significantly on $25^{\circ} \mathrm{C}$ whereas on $5 \pm 3^{\circ} \mathrm{C}$ slight change in these parameters has been observed. During the storage time, no visual color change was noticed in any sample. The decrease of entrapment efficiency and increase in particle size of nanoparticles might be attributed to the semi-amorphous nature of the SAF127 copolymer in MN3. When lipophilic part of copolymer exposed to temperature or light (kinetic energy), semi-amorphous state changes into the more stable amorphous state which leads to increase in particle size and expulsion of entrapped drug from the polymeric matrix [13]. The nanoparticles stored at $5 \pm 3^{\circ} \mathrm{C}$ showed slight deviation in studied parameters, which suggested that the above temperature was optimum storage temperature.

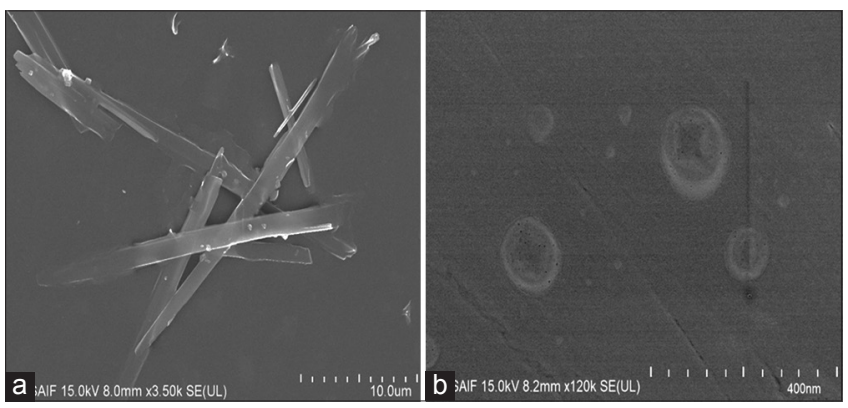

Fig. 6: Scanning electron microscopy image of (a) pure metformin and (b) MN3

\section{CONCLUSION}

MNs were successfully prepared by emulsion solvent evaporation technique using SAF127 copolymer and PVA. The drug showed no compatibility issues with excipients. Particle size, PDI, zeta potential, drug encapsulation efficiency, and percentage yield of MNs were found to be influenced by copolymer concentration. The SAF127 copolymer contains hydrophilic and hydrophobic portions which encapsulated metformin and produces nano-sized particles. In vitro release of metformin from nanoparticles was significantly prolonged to $30 \mathrm{~h}$ compared to pure drug. Further, to explore the application of SAF127 copolymer as a nanocarrier for oral metformin delivery clinical studies is required. 


\section{AUTHOR'S CONTRIBUTION}

VKK has performed and wrote the manuscript. PKV has designed, supervised, and checked the results. All authors read and approved the final manuscript.

\section{ACKNOWLEDGMENT}

The authors are grateful to University Grant Commission (UGC), New Delhi, for providing fellowship under Major Research Project (MRP) Reference No: FN/42/703/2013/SR. Authors are grateful to Dr. Munish Ahuja and Dr. Anil Ohlan for providing facilities for Zetasizer and XRD, respectively.

\section{CONFLICTS OF INTEREST}

All the authors hereby declare that there are no conflicts of interest.

\section{REFERENCES}

1. Mishra MK, Ray D, Barik BB. Microcapsules and transdermal patch: A comparative approach for improved delivery of antidiabetic drug. AAPS Pharm Sci Tech 2009;10: 928-34.

2. Barwal I, Sood A, Sharma M, Singh B, Yadav SC. Development of stevioside Pluronic F-68 copolymer based PLA-nanoparticles as an antidiabetic nanomedicine. Colloids Surfaces B Biointerfaces 2013;101:510-16.

3. El-Gendy N, Hosny A, Abdelbary A. Microencapsulation approach for orally extended delivery of glipizide: In vitro and in vivo evaluation. Indian J Pharm Sci 2012;74:319-30.

4. Sankhyan A, Pawar PK. Metformin loaded non-ionic surfactant vesicles: Optimization of formulation, effect of process variables and characterization. DARU J Pharm Sci 2013;21: 7.

5. Kai S, Huang L, Zhao Y, Guo S. Polymeric metformin and its use as a therapeutic agent and as a delivery vehicle. WO 2016144766 A1.

6. Rani R, Dahiya S, Dhingra D, Dilbaghi N, Kim KH, Kumar S. Evaluation of anti-diabetic activity of glycyrrhizin-loaded nanoparticles in nicotinamide-streptozotocin-induced diabetic rats. Eur J Pharm Sci 2017;06:220-30

7. Mohamed MN, Khaleid MA, Mohamed S. Formulation and evaluation of extended release metformin hydrochloride beads. Int J Pharm Pharm Sci 2014;6:433-41

8. Monther FM, Inam SA, Najwan KJ. Design, synthesis and preliminary pharmacological evaluation of new metformin derivatives. Int J Pharm Pharm Sci 2017;9:279-93.

9. Corti G, Capasso G, Maestrelli F, Cirri M, Mura P. Physical-chemical characterization of binary systems of metformin hydrochloride with triacetyl- $\beta$-cyclodextrin. J Pharm Biomed Anal 2007;45:480-86.

10. Verma P, Kamboj V. Synthesis and antidiabetic activity of N'-[3-(alkyl/ aryl substituted)-4-oxo-1,3-thiazolidin-2-yleidene-2-(pyrazin-2yloxy)acetohydrazide. Acta Pharma Sci 2010;52:411-15.

11. Javidfar S, Pilehvar-Soltanahmadi Y, Farajzadeh R, Lotfi-Attari J, Shafiei-Irannejad V, Hashemi M, et al. The inhibitory effects of nanoencapsulated metformin on growth and hTERT expression in breast cancer cells. J Drug Deliv Sci Technol 2017;43:19-26.

12. Kamboj VK, Verma PK. Poloxamers based nanocarriers for drug delivery system. Der Pharma Lett 2015;7:264-69.

13. Elbahwy IA, Ibrahim HM, Ismael HR, Kasem AA. Enhancing bioavailability and controlling the release of glibenclamide from optimized solid lipid nanoparticles. J Drug Deliv Sci Technol 2017;38:78-89.

14. Lu Y, Park K. Polymeric micelles and alternative nanosized delivery vehicles for poorly soluble drugs. Int J Pharm 2013;453:198-14

15. Gao Q, Liang Q, Yu F, Xu J, Zhao Q, Sun B. Synthesis and characterization of novel amphiphilic copolymer stearic acid-coupled F127 nanoparticles for nano-technology based drug delivery system. Colloids Surfaces B Biointerfaces 2011;88:741-48.

16. Shariatinia Z, Zahraee Z. Controlled release of metformin from chitosan-based nanocomposite films containing mesoporous MCM-41 nanoparticles as novel drug delivery systems. J Colloid Interface Sci 2017;501:60-76

17. Kumar S, Bhanjana G, Verma RK, Dhingra D, Dilbaghi N, Kim KH. Metformin-loaded alginate nanoparticles as an effective anti-diabetic agent for controlled drug release. J Pharm Pharmacol 2017;69:143-50.

18. Lokhande AB, Mishra S, Kulkarni RD, Naik JB. Influence of different viscosity grade ethylcellulose polymers on encapsulation and in vitro release study of drug-loaded nanoparticles. J Pharm Res 2013;7:414-20.

19. Prakash C, Kamboj VK, Ahlawat, P, Kumar V. Structural and molecular alterations in arsenic-induced hepatic oxidative stress in rats: A FTIR study. Toxicol Environ Chem 2015;97:1408-21.

20. Snima KS, Jayakumar R, Lakshmanan VK. In vitro and in vivo biological evaluation of O-carboxymethyl chitosan encapsulated metformin nanoparticles for pancreatic cancer therapy. Pharm Res 2014;31:3361-70.

21. Hu LD, Liu Y, Tang X, Zhang Q. Preparation and in vitro/in vivo evaluation of sustained-release metformin hydrochloride pellets. Eur J Pharm Biopharm 2006;64:185-92.

22. Patil P, Bhoskar M. Optimization and evaluation of spray dried chitosan nanoparticles containing doxorubicin. Int J Curr Pharm Res 2014;6:7-15

23. Birnbaum DT, Kosmala JD, Brannon-Peppas L. Optimization of preparation techniques for poly(lactic acid-co-glycolic acid) nanoparticles. J Nano Res 2000:2:173-81.

24. Jain S Saraf S. Influence of processing variables and in vitro characterization of glipizide loaded biodegradable nanoparticles. Diabetes Metab Syndr Clin Res Rev 2009;3: 113-17.

25. Kusum VD, Bhosale UV. Formulation and optimization of polymeric nano drug delivery system of acyclovir using 32 full factorial design. Int J Pharm Tech Res 2009;1:644-53.

26. Mokale V, Rajput R, Patil J, Yadava S, Naik J. Formulation of metformin hydrochloride nanoparticles by using spray drying technique and in vitro evaluation of sustained release with 32-level factorial design approach. Dry Technol 2016;34:1455-61.

27. Domingos RF, Baalousha MA, Ju-Nam Y, Reid MM, Tufenkji N, Lead JR, et al. Characterizing manufactured nanoparticles in the environment: Multimethod determination of particle sizes. Environ Sci Technol 2009;43:7277-84 